\title{
Mejora del Proceso de Enseñanza y Aprendizaje en la Carrera de Ingeniería de Ejecución Mecánica. Diseño Micro-curricular Basado en Resultados de Aprendizaje
}

\author{
Alessandro R. Avagliano y Sylvana A. Vega \\ Departamento de Ingeniería Mecánica, Universidad de Santiago de Chile, Av. Libertador Bernardo \\ O'Higgins 3363, Estación Central, Santiago, Chile. (e-mail: alessandro.avagliano@usach.cl; \\ silvana.vega@usach.cl)
}

Recibido Ene. 16, 2013; Aceptado Feb. 13, 2013; Versión final recibida Abr. 10, 2013

\begin{abstract}
Resumen
El presente trabajo corresponde a una investigación cuasi experimental centrada en el diseño microcurricular en el marco de un mejoramiento del proceso de enseñanza aprendizaje en dos asignaturas de la carrera de Ingeniería de Ejecución Mecánica de la Universidad de Santiago de Chile. Para el estudio se adquirió e implementó un Aula Digital Interactiva y se consideró un grupo experimental y un grupo de control en la asignatura de Formulación de Proyectos y un caso de estudio cualitativo para la asignatura de Sistemas Térmicos. En ambos cursos se diseñaron los programas de estudios, basándose en resultados de aprendizaje, perfil de egreso y modelo educativo institucional. Los resultados muestran que se mejoró el rendimiento académico en un 20\% del grupo experimental en comparación al grupo de control que usó el diseño curricular vigente. Esto permitirá modificar las estrategias de enseñanza, obteniendo una mayor equidad académica entre los diferentes modos personales de aprender.
\end{abstract}

Palabras clave: investigación educativa, diseño micro-curricular, aula digital interactiva, enseñanzaaprendizaje

\section{Improved Teaching and Learning Process in the Mechanical Engineering Career. Micro-curricular Redesign Based on Learning Outcomes}

\begin{abstract}
This work corresponds to a quasi-experimental research focused on micro-curricular design in the context of improving the teaching-learning process in two subjects of the mechanical engineering career of the Universidad de Santiago de Chile. For the study, an Interactive Digital Classroom was acquired and implemented and an experimental group and a control group in the course of Projects Formulation and a qualitative case study to the subject of Thermal Systems were considered. In both courses the curricular programs based on learning outcomes, graduate profile and institutional educational model were designed. The results show that academic performance improved by $20 \%$ in the experimental group compared to the control group that used the current curricular design. This will allow modifying teaching strategies and achieving greater academic equity between different personal ways of learning.
\end{abstract}

Keywords: educational research, micro-curricular design, interactive digital classroom, teaching-learning 


\section{INTRODUCCIÓN}

En el marco del aseguramiento de la calidad en la educación superior es imprescindible la mejora continua del proceso enseñanza aprendizaje en las asignaturas de los programas de estudios. Esta mejora involucra un rediseño de los programas de las asignaturas donde los resultados de aprendizaje, perfil de egreso y Modelo Educativo Institucional juegan un rol importante en este proceso de enseñanza aprendizaje centrada en los estudiantes (Rué, 2007). Sin embargo, esta mejora debe acompañarse de un cambio en los paradigmas del profesor, donde la labor docente se transforma de un transmisor a un facilitador de la construcción del conocimiento, reflexionando constantemente la práctica docente (Prieto, 2007; Gomes, 2002).

El rediseño a nivel microcurricular, es decir del programa de la asignatura impartida, incluye la adaptación de los contenidos al uso de tecnologías, como herramientas facilitadoras en el proceso enseñanza aprendizaje centrado en el estudiante.

Aunque numerosos estudios, a nivel primario, secundario y educación superior señalan la relevancia de la implementación para el proceso formativo del aprendizaje nuevas tecnologías y modelos que permitan el mejoramiento continuo del resultado del aprendizaje (Cataldi et al., 2006; Candanedo, 2005; Balmaceda et al., 2006; González, 2006; Guzmán et al., 2006), en el campo de la ingeniería mecánica esto se refleja con mayor lentitud, sobre todo lo que involucra el rediseño microcurricular de los programas de las asignaturas de los planes de estudio de las carreras.

Estas nuevas tecnologías se enmarcan en las denominadas TIC (Tecnologías de la Información y Comunicación), las cuales se transforman en un elemento innovador de ayuda a la docencia, ya que permiten un cambio de mentalidad o paradigma en el docente, centrando el foco de enseñanza en el estudiante (González, 2006 ).

Asimismo, Cabero, 2000, señala que estas tecnologías generan espacios de aprendizaje superiores al de una comunicación presencial, ya que generan entornos colaborativos de aprendizaje, donde los estudiantes se convierten en los constructores de la información. Sin embargo, esto debe ser acompañado por un cambio en los paradigmas del profesor, el cual debe desempañar un nuevo rol como consultor, moderador, facilitador de la información y del aprendizaje, pasando de "ser un experto en contenidos a un facilitador del aprendizaje". Esto significa que el docente deberá diseñar las experiencias de aprendizaje, generar la interacción inicial de los estudiantes, motivar el autoestudio, la colaboración entre pares y ayudar a los estudiantes con sus habilidades.

De este modo, Salinas, 2004, propone tres enfoques principales en torno a la identificación de una institución y el proceso de adaptación de enseñanza aprendizaje a través de TIC, con un determinado foco de calidad: "Un enfoque tecnológico, que está basado en la idea de que la sofisticación del entorno tecnológico y la calidad del proceso enseñanza - aprendizaje, son directamente proporcionales, con lo que, a mayor sofisticación técnica, mayor calidad. El contenido es lo importante, donde se basa la calidad del proceso enseñanza - aprendizaje en los contenidos docentes, es decir, la reelaboración de la información que los docentes hacen en los materiales y su mejor uso y distribución se considera la base de la calidad. En este caso, cuanto más sofisticados sean los materiales, mayor será la calidad del proceso. Un enfoque metodológico que se centra en el alumno y que, partiendo de criterios pedagógicos, basa la calidad en una adecuada combinación en cada caso de decisiones que tienen que ver con la tecnología a utilizar, con la función pedagógica que el entorno cumplirá y con los aspectos de organización del proceso dentro de dicho entorno."

En la literatura se señalan variados ejemplos de la aplicación de tecnologías en el aula, tal como lo indica Villareal, 2006, acerca del uso de una Pizarra Digital Interactiva. El autor muestra que esta tecnología logra un trabajo colaborativo, desarrollando autonomía en el aprendizaje y construcción de conocimientos. Además, permite una interacción continua profesor- estudiante, ya que se facilita la flexibilidad en el desarrollo del contenido de la clase, permitiendo la modificación de la sesión de acuerdo a las necesidades de los estudiantes. "Por medio de las preguntas y respuestas con los alumnos, el profesor puede dirigir el trabajo, según las reacciones de sus alumnos, pudiendo también manejar de mejor manera los avances individuales a partir de la respuesta de cada alumno" (Villareal, 2006).

La ventaja de un Aula Digital Interactiva (ADI), es que permite que los estudiantes capten con mayor facilidad el contenido de las asignaturas, ya que permite el acceso de material audiovisual, donde el profesor maneja una pantalla de computador desde la propia pizarra y las anotaciones manuscritas en ella son guardadas y enviadas por correo electrónico a todos los estudiantes, tanto presentes en el aula como los que no hayan podido asistir a clase. Además, la incorporación de un sistema de votación interactiva, 
conocida en el mercado como tecleras digitales, conlleva a una evaluación formativa en la clase, donde el profesor construye actividades con preguntas que el estudiante deberá responder, permitiendo que los contenidos sean revisados in situ conforme al estilo de aprendizaje de cada estudiante (Martínez, 2007).

El presente trabajo tiene como objetivo mejorar el proceso de enseñanza aprendizaje en las asignaturas Sistemas Térmicos y Formulación de Proyectos, incorporando un Aula Digital Interactiva, en el marco de un modelo de innovación curricular, donde se espera realizar un rediseño microcurricular en las asignaturas mencionadas aplicando la(s) estrategia(s) de aprendizaje adecuada(s), implementar un Aula Digital Interactiva en el Departamento de Ingeniería Mecánica acorde a las necesidades académicas de dichos cursos pertenecientes al plan de estudio de la carrera de Ingeniería de Ejecución Mecánica, evaluar el mejoramiento del proceso enseñanza y aprendizaje utilizando el Aula Digital Interactiva y mejorar el rendimiento académico de los estudiantes de las asignaturas Sistemas Térmicos y Formulación de Proyectos, utilizando Tecnologías de la Información y Comunicación (TIC).

\section{METODOLOGÍA}

El presente estudio se centra en el rediseño microcurricular de los programas de las asignaturas de Sistemas Térmicos y Formulación de Proyectos, correspondientes al plan de estudio vigente de la carrera de Ingeniería de Ejecución Mecánica de los últimos niveles. Además, se implementa un Aula Digital Interactiva $(A D I)$ en el Departamento de Ingeniería Mecánica, como parte de la investigación que se presenta.

\section{Rediseño microcurricular}

Como parte del rediseño microcurricular de las asignaturas de Sistemas Térmicos y Formulación de Proyectos se comienza con una reflexión de la práctica docente y la incorporación de resultados de aprendizaje en el proceso de enseñanza de las asignaturas antes mencionadas. Esto permite conocer las deficiencias que presenta la enseñanza de estos cursos con los objetivos planteados en el programa de las asignaturas y una mejora en la docencia en aula.

Luego se revisan y reformulan los objetivos de los programas de las asignaturas estudiadas, transformándolos en resultados de aprendizaje que sean coherentes con el Modelo Educativo Institucional de la Universidad de Santiago de Chile y perfil de egreso de la carrera de Ingeniería de Ejecución en Mecánica. De esta forma, se alinea el estudio en torno al proyecto educativo de la Universidad que se encuentra centrado en la calidad, la flexibilidad y el aprendizaje. Aumentando la satisfacción de los estudiantes con una propuesta de valor, acentuando el desarrollo académico y con una clara responsabilidad social basada en la innovación tecnológica y del conocimiento. Cabe señalar, que el proyecto educativo institucional está dirigido a estudiantes que mayoritariamente provienen de un estrato socioeconómico que les ha impedido acceder a una educación primaria y secundaria de excelencia, causándoles dificultades de aprendizaje y bajos niveles de conocimientos para la educación superior.

A continuación, se establecen estándares curriculares y de desempeño para cada uno de los resultados de aprendizaje esperados, los cuales se definen para cada una de las asignaturas estudiadas.

De este modo, se desarrolla el rediseño microcurricular en las asignaturas, aplicando estrategia(s) de aprendizaje adecuada(s), una planificación curricular adaptada al uso del Aula Digital Interactiva y técnicas apropiadas de evaluación en cada una de las asignaturas. Posteriormente, se elaboran instrumentos para el seguimiento, la observación y la recopilación de información. Por ejemplo, en Formulación de Proyectos se aplica una pre-prueba tipo diagnóstico que permita señalar el nivel de conocimiento, el test de CHAEA (Cuestionario Honey y Alonso de Estilos de Aprendizaje. Martínez, 2007) para identificar el estilo de aprendizaje de cada estudiante, pruebas parciales y pruebas de reemplazo. Conjuntamente, en Sistemas Térmicos se miden los cambios con un test de diagnóstico, test de CHAEA, registro anecdótico en aula y encuestas que permitan observar la motivación de los estudiantes.

\section{Implementación del Aula Digital Interactiva (ADI)}

La implementación del Aula Digital Interactiva (ADI) en el Departamento de Ingeniería Mecánica permite focalizar el proceso formativo en el aprendizaje del estudiante, cumpliendo con el perfil de egreso, cambiar el paradigma académico de los profesores involucrados en el proyecto y mejorar el proceso de enseñanza aprendizaje en las asignaturas Sistemas Térmicos y Formulación de Proyectos. Además, es posible modificar microcurricularmente las asignaturas antes mencionadas, basándose en el logro de resultados de aprendizaje adaptados al uso de la tecnología y establecer una planificación curricular de los cursos estudiados, considerando estrategias de enseñanza que involucren la implementación del ADI. 
EI ADI consta de los siguientes elementos, tal como se ilustra en la Figura 1: Una sala equipada con mobiliario cómodo, pizarra y luminosidad regulada por persianas adecuadas para la visualización correcta de la Pizarra Digital Interactiva, Pizarra Digital Dualboard Interwrite, Tecleras Digitales de Votación Electrónica CPS IR, computador, conexión a Internet, proyector multimedia y equipo de audio conectado a los equipos computacionales.

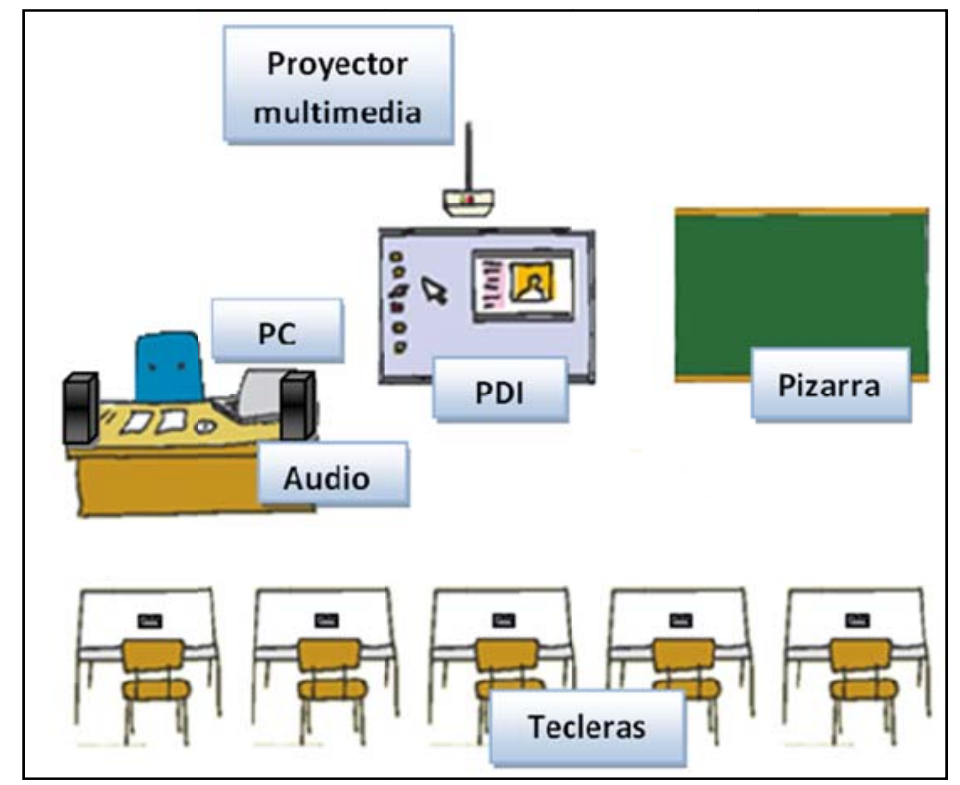

Fig. 1: Elementos del Aula Digital Interactiva

Un ejemplo de aplicación del ADI en la asignatura de Sistemas Térmicos es el siguiente: al estudiar la unidad de generadores de vapor se plantea un esquema virtual, conceptual y básico de un generador de vapor, los estudiantes deben desplazar con el lápiz de la pizarra digital algunos íconos de equipos térmicos que mejoren la eficiencia energética del generador. Luego, el profesor hace una revisión in situ de las soluciones planteadas por los estudiantes. En seguida, para evaluar el aprendizaje de esta unidad se plantea una pregunta de selección múltiple al curso, la cual se responde a través de las tecleras digitales de votación electrónica en un tiempo definido por el profesor. Finalmente, se presenta en la pizarra un histograma el cual indica las alternativas seleccionadas por los estudiantes y la alternativa correcta. Con esta información el profesor realiza una retroalimentación del contenido de la unidad estudiada. Si se hubiese enseñado con el modelo tradicional de la pizarra clásica, el tiempo para desarrollar el mismo ejercicio sería mayor, ya que los estudiantes tendrían que dibujar cada uno de los equipos, restando tiempo para evaluaciones formativas.

\section{DESARROLLO DEL ESTUDIO}

El trabajo se basa, por una parte, en una investigación cuasi-experimental, dado que se utiliza un grupo experimental $\left(2^{\circ}\right.$ semestre 2010) y un grupo de control ( $1^{\circ}$ semestre 2010$)$, para la asignatura de Formulación de Proyectos y por otro lado será una investigación basada en un caso de estudio donde se presenta en forma cualitativa el análisis de las variables en la asignatura de Sistemas Térmicos, dado que se desarrolla dentro de un semestre $\left(2^{\circ}\right.$ semestre 2010$)$.

En la primera etapa, se informa y motiva a los estudiantes para el desarrollo de las nuevas actividades y formas de interactuar en el aula, dándoles a conocer la relevancia del estudio y lla importancia que ellos tienen en éste. En ambas investigaciones se aplica a todos los grupos en estudio un test de diagnóstico y el test de CHAEA.

En particular, la asignatura de Formulación de Proyectos trabaja con el grupo experimental utilizando el ADI, mientras que en el grupo de control la enseñanza se realiza de la manera tradicional, sin el uso de TIC. La metodología tradicional incluye la utilización de la pizarra blanca, con plumones, es básicamente expositiva, sin aplicación del test de CHAEA, ni test de diagnóstico, sólo pruebas parciales y de reemplazo como instrumentos de evaluación. En cambio en el grupo experimental se incluyen metodologías activas de enseñanza, que abarcan todos los estilos de aprendizaje de los estudiantes. En este grupo se utiliza el ADI tanto en cátedra como ayudantía, aplicándose todos los instrumentos de evaluación antes mencionados. 
Por otra parte, en la asignatura de Sistemas Térmicos se trabaja en forma cualitativa con el grupo curso, tanto en cátedra como en ayudantía, donde en forma alternada se utiliza el ADI. Esto permite evidenciar en los estudiantes la motivación, las impresiones y la efectividad en términos del aprendizaje mediante cuatro encuestas realizadas durante el semestre, las cuales contemplaron cuatro aspectos evaluados: ¿Cómo se sienten y relacionan los estudiantes con las nuevas tecnologías y el rediseño del programa de la asignatura? ¿Cuál es la conexión entre el aprendizaje y la participación del estudiante cuando utiliza la pizarra interactiva digital, comparándola con el método tradicional de enseñanza pizarra y plumón? ¿Cuál es la percepción del estudiante cuando se utiliza la pizarra digital interactiva? ¿Cuál es la opinión del estudiante en relación a la eficacia de la enseñanza del profesor cuando se utiliza un aula interactiva digital? Además, para efectos de comparación en los estilos de aprendizaje de los estudiantes, se utilizan los resultados que arrojó el test de CHAEA aplicado en el curso. También se aplica un registro anecdótico durante el semestre en estudio, para obtener información respecto al comportamiento de los alumnos con el uso de los recursos tecnológicos.

Al final de la etapa de implementación se aplica una prueba parcial en Formulación de Proyectos y el test de CHAEA en ambas asignaturas de la presente investigación, con la finalidad de observar los cambios en los estilos de aprendizajes y los conocimientos alcanzados por cada grupo para compararlos y medir el efecto de la utilización del ADI. Por último, se procede a evaluar la información recolectadia del período de estudio de ambas asignaturas. Con estos datos se elaboran estadísticas que permitan mostrar la mejora del proceso enseñanza- aprendizaje y el aumento del rendimiento académico de los estudiantes, utilizando el ADI.

\section{RESULTADOS DE LA INVESTIGACIÓN}

Se implementa el Aula Digital Interactiva (ADI) en la sala n 210 del Departamento de Ingeniería Mecánica de la Universidad de Santiago de Chile. Esto permite una remodelación integral de la misma, que consiste en: ventanas con aislación acústica, cortinas de control de iluminación, data show, pizarra digital interactiva, pizarra blanca tradicional, sistema de audio, nuevo alfombrado, computador y escritorio modular para el profesor. Estos cambios fueron orientados con el fin de obtener un ambiente apropiado para mejorar el proceso de enseñanza aprendizaje. Cabe señalar que algunos estudiantes expresaron verbalmente al docente que se sentían cómodos en la nueva sala. Este cambio se muestra en la Figura 2.

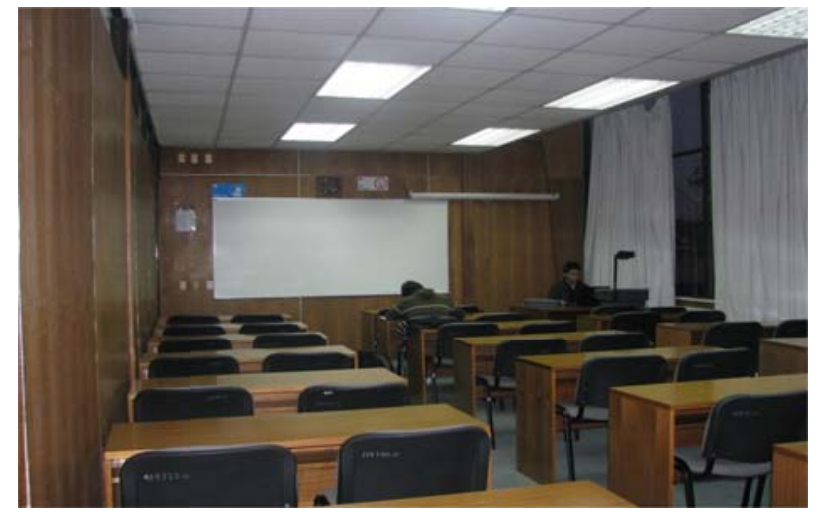

a.

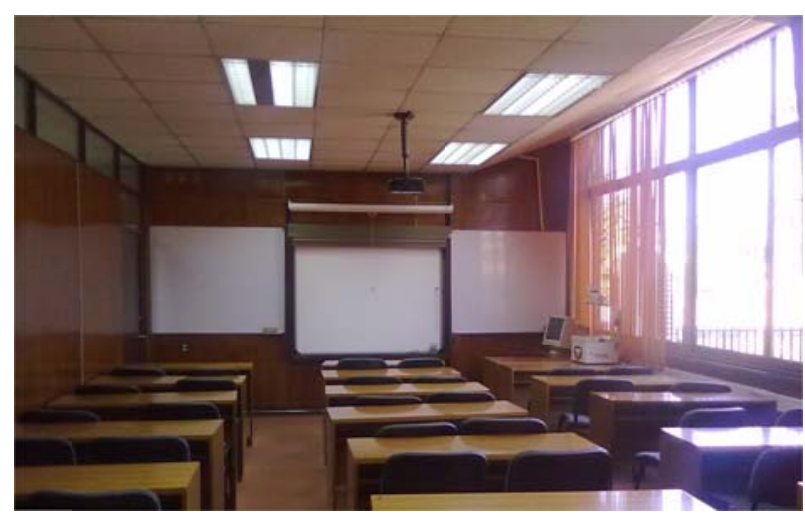

b.

Fig. 2: Implementación de la sala $n^{\circ}$ 210. a) Antes. b) Después.

Se desarrollan programas de estudios rediseñados de las asignaturas basados en: resultados de aprendizaje, perfil de egreso y Modelo Educativo Institucional. Además, se mejora el proceso de enseñanza aprendizaje al conocer el estilo de aprendizaje de los estudiantes mediante la aplicación del test de CHAEA, el cual se entregó a cada estudiante y, una vez desarrollado, finalizan sumando el puntaje total de cada estilo de aprendizaje. Con los resultados obtenidos se determinó el estilo de aprendizaje dominante de cada uno y se calculó porcentualmente en base al total de números de estudiantes de cada curso, observando el cambio que hubo antes y después del presente estudio, tal como se ilustra en la Figura 3. En el caso de la asignatura de Formulación de Proyectos, el test de CHAEA se aplicó al finalizar en cada semestre, en cambio en el curso de Sistemas Térmicos, se midió al inicio y término del semestre. Los resultados del test permitieron variar las estrategias de enseñanza con el fin de obtener una mayor equidad académica entre los diferentes modos personales de aprender. Esto se observa en la Figura 3, donde en el curso de Formulación de Proyectos se refleja un aumento del estilo de aprendizaje reflexivo, teórico y pragmático, ya que se realizaron actividades en el aula que fomentaron este cambio, autoevaluaciones informales, 
planteamientos de problemas abiertos reales y virtuales, construcción de hipótesis para resolver problemas, uso de la pizarra interactiva en actividades de evaluación formativa, entre otras. En cambio en la asignatura de Sistemas Térmicos, hubo una disminución de los estilos activo, teórico y pragmático, aumentando significativamente el perfil reflexivo, pues hubo un cambio de profesor en último momento y el estilo de aprendizaje predominante de éste era reflexivo. Esto muestra la influencia que tiene el estilo de aprendizaje del docente en su enseñanza.

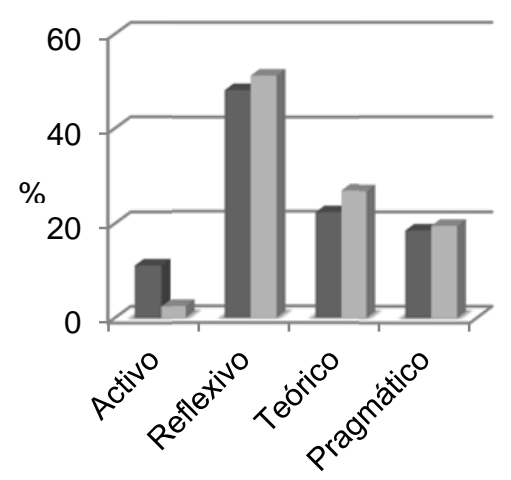

a.
Grupo control
$\left(1^{\circ}\right.$ sem $)$

Grupo experimental $\left(2^{\circ} \mathrm{sem}\right)$

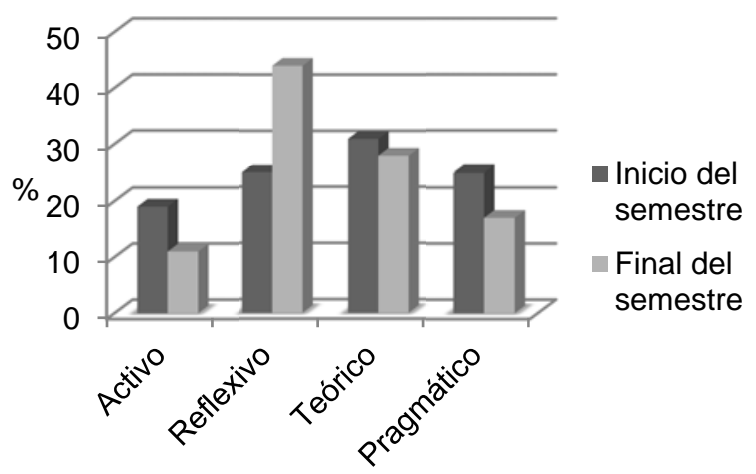

b.

Fig. 3: Comparación del porcentaje de estudiantes que presentan un perfil dominante de aprendizaje, evaluado por el test CHAEA de las asignaturas estudiadas. a) Formulación de Proyectos b) Sistemas Térmicos

Se puede apreciar en la Tabla 1 una gran diferencia entre los porcentajes de cada categoría de notas de la asignatura de Formulación de Proyectos, donde la mayor cantidad de estudiantes se ubican con notas de aprobación entre 4,0 y 4,9. Si se considera que la nota mínima de aprobación definida por la Universidad es igual o superior a 4,0, incluyendo la Prueba Optativa de Reemplazo (POR), se observa en los resultados que el $71,43 \%$ de los estudiantes del grupo de control y el $93,85 \%$ de los del grupo experimental aprobaron. Lo que se relaciona con la tendencia levemente superior en las dimensiones teórica y reflexiva del curso en estudio. En los resultados también se aprecia que el curso experimental constituido por 45 alumnos tuvo mejores resultados que el grupo de control compuesto sólo por 14 alumnos, lo que muestra que la estrategia y didáctica aplicada por el profesor influye preponderantemente en los resultados obtenidos de los estudiantes. Aunque los resultados de los promedios y la desviación estándar del grupo de control y grupo experimental son similares, los resultados muestran que, analizando la aprobación del curso excluyendo la prueba POR, el $63,29 \%$ de los estudiantes del grupo de control y el $84,45 \%$ de los del grupo experimental aprobaron. Esto implica que el uso del ADI favoreció el proceso de enseñanza aprendizaje. Las calificaciones promedios y desviación estándar se muestran en las Tablas 2 y 3, respectivamente.

Tabla 1: Comparación de notas finales de la asignatura de Formulación de Proyectos entre el grupo control (1er semestre) y el grupo experimental (2do semestre), con y sin la Prueba Optativa de Reemplazo (POR)

\begin{tabular}{|c|c|c|c|c|}
\hline \multicolumn{5}{|c|}{ 1er Semestre } \\
\hline $\begin{array}{c}\text { Notas } \\
\text { Promedio }\end{array}$ & $\mathrm{N}^{\circ}$ alumnos s/POR & $\%$ curso s/POR & $\mathrm{N}^{\circ}$ alumnos c/POR & $\%$ curso c/POR \\
\hline $1,0-2,9$ & 0 & 0 & 0 & 0 \\
\hline $3,0-3,9$ & 5 & 35,71 & 4 & 28,57 \\
\hline $4,0-4,9$ & 8 & 57,14 & 9 & 64,29 \\
\hline $5,0-5,9$ & 1 & 7,15 & 1 & 7,14 \\
\hline $6,0-7,0$ & 0 & 0 & 0 & 0 \\
\hline \multicolumn{5}{|c|}{ 2do Semestre } \\
\hline $\begin{array}{c}\text { Notas } \\
\text { Promedio }\end{array}$ & $\mathrm{N}^{\circ}$ alumnos s/POR & $\%$ curso s/POR & $\mathrm{N}^{\circ}$ alumnos c/POR & $\%$ curso c/POR \\
\hline $1,0-2,9$ & 1 & 2,22 & 1 & 2,22 \\
\hline $3,0-3,9$ & 6 & 13,33 & 2 & 4,44 \\
\hline $4,0-4,9$ & 36 & 80 & 40 & 88,89 \\
\hline $5,0-5,9$ & 2 & 4,45 & 2 & 4,46 \\
\hline $6,0-7,0$ & 0 & 0 & 0 & 0 \\
\hline
\end{tabular}

En la asignatura de Sistemas Térmicos, los promedios de los aspectos más y menos valorados en las encuestas se muestran en la tabla 4, donde se califica la pregunta con una nota de 1 a 4, siendo 1 la valoración más desfavorable y 4 la valoración más favorable. 
Tabla 2: Calificaciones promedios de la asignatura de Formulación de Proyectos entre el grupo experimental y el grupo control.

\begin{tabular}{|c|c|c|}
\hline Media Promedio & Pruebas escritas programadas & Con Prueba Optativa de Reemplazo \\
\hline 1er semestre & 4,16 & 4,24 \\
\hline 2do semestre & 4,31 & 4,39 \\
\hline
\end{tabular}

Tabla 3: Desviación estándar promedio de la asignatura de Formulación de Proyectos entre el grupo experimental y el grupo control.

\begin{tabular}{|c|c|c|}
\hline DE promedios & Pruebas escritas programadas & Con Prueba Optativa de Reemplazo \\
\hline 1er semestre & 0,61 & 0,58 \\
\hline 2do semestre & 0,55 & 0,48 \\
\hline
\end{tabular}

Tabla 4: Promedios de los aspectos más y menos valorados en las encuestas de la asignatura de Sistemas Térmicos.

\begin{tabular}{|c|c|c|c|c|}
\hline Aspectos evaluados & Prom. & Valoración & Descripción de la pregunta & $\begin{array}{l}\text { Promedio } \\
\text { pregunta }\end{array}$ \\
\hline \multirow{4}{*}{$\begin{array}{l}\text { Apreciación y } \\
\text { relación de los } \\
\text { estudiantes con las } \\
\text { nuevas tecnologías y } \\
\text { el rediseño del } \\
\text { programa de la } \\
\text { asignatura }\end{array}$} & \multirow{4}{*}{2,7} & \multirow{2}{*}{$\begin{array}{l}\text { Más } \\
\text { valorados }\end{array}$} & $\begin{array}{l}\text { Me gustaría que los cambios en los objetivos y } \\
\text { contenidos del curso se realizaran también en otras } \\
\text { asignaturas }\end{array}$ & 3,0 \\
\hline & & & $\begin{array}{l}\text { El profesor enseña los contenidos de manera más } \\
\text { rápida y fácil con el uso de los equipos tecnológicos } \\
\text { implementados en el aula }\end{array}$ & 2,8 \\
\hline & & \multirow{2}{*}{$\begin{array}{l}\text { Menos } \\
\text { valorados }\end{array}$} & $\begin{array}{l}\text { Los cambios en los objetivos y contenidos del curso } \\
\text { han ayudado a mejorar la eficacia de la enseñanza } \\
\text { del profesor }\end{array}$ & 2,5 \\
\hline & & & $\begin{array}{l}\text { Mi experiencia general con los cambios en los } \\
\text { objetivos y contenidos del curso ha sido positiva }\end{array}$ & 2,4 \\
\hline \multirow{4}{*}{$\begin{array}{l}\text { Conexión entre el } \\
\text { aprendizaje y la } \\
\text { participación del } \\
\text { estudiante cuando } \\
\text { utiliza la pizarra } \\
\text { interactiva digital, } \\
\text { comparándola con el } \\
\text { método tradicional de } \\
\text { enseñanza pizarra y } \\
\text { plumón }\end{array}$} & \multirow{4}{*}{1,3} & \multirow[t]{2}{*}{$\begin{array}{l}\text { Más } \\
\text { valorados }\end{array}$} & $\begin{array}{l}\text { El uso de la pizarra interactiva permite, en general, } \\
\text { comprender mejor los contenidos del curso que con } \\
\text { el sistema convencional de enseñanza (pizarra } \\
\text { blanca-plumón) }\end{array}$ & 1,4 \\
\hline & & & $\begin{array}{l}\text { La pizarra interactiva facilita trabajar diagramas, } \\
\text { tablas, figuras y/o gráficos }\end{array}$ & 1,4 \\
\hline & & \multirow{2}{*}{$\begin{array}{l}\text { Menos } \\
\text { valorados }\end{array}$} & $\begin{array}{l}\text { La pizarra interactiva facilita la presentación visual } \\
\text { de los contenidos del curso }\end{array}$ & 1,2 \\
\hline & & & $\begin{array}{l}\text { La pizarra interactiva permite una clase más } \\
\text { interactiva y dinámica }\end{array}$ & 1,1 \\
\hline \multirow{4}{*}{$\begin{array}{l}\text { Percepción del } \\
\text { estudiante cuando se } \\
\text { utiliza la pizarra } \\
\text { digital interactiva }\end{array}$} & \multirow{4}{*}{1,4} & \multirow{2}{*}{$\begin{array}{l}\text { Más } \\
\text { valorados }\end{array}$} & $\begin{array}{l}\text { El tiempo de las clases se redujeron con el uso de la } \\
\text { pizarra interactiva }\end{array}$ & 1,5 \\
\hline & & & $\begin{array}{l}\text { Me gustaría que la pizarra digital interactiva } \\
\text { estuviese disponible para su uso en otras } \\
\text { asignaturas }\end{array}$ & 1,4 \\
\hline & & \multirow{2}{*}{$\begin{array}{l}\text { Menos } \\
\text { valorados }\end{array}$} & $\begin{array}{l}\text { El profesor hace uso de la pizarra interactiva para } \\
\text { introducir el tema de la clase }\end{array}$ & 1,4 \\
\hline & & & $\begin{array}{l}\text { El profesor hace uso de la pizarra interactiva para } \\
\text { cerrar la sesión de la clase }\end{array}$ & 1,4 \\
\hline \multirow{4}{*}{$\begin{array}{l}\text { Opinión del } \\
\text { estudiante en } \\
\text { relación a la eficacia } \\
\text { de la enseñanza del } \\
\text { profesor cuando se } \\
\text { utiliza un aula } \\
\text { interactiva digital }\end{array}$} & \multirow{4}{*}{2,8} & \multirow{2}{*}{$\begin{array}{l}\text { Más } \\
\text { valorados }\end{array}$} & $\begin{array}{l}\text { El uso de equipos tecnológicos en el aula facilita el } \\
\text { logro de los objetivos propuestos al comienzo de la } \\
\text { asignatura }\end{array}$ & 3,1 \\
\hline & & & $\begin{array}{l}\text { Mi experiencia general con la implementación de un } \\
\text { aula interactiva digital ha sido positiva }\end{array}$ & 2,9 \\
\hline & & \multirow[b]{2}{*}{$\begin{array}{l}\text { Menos } \\
\text { valorados }\end{array}$} & $\begin{array}{l}\text { El uso de una pizarra interactiva digital me ha } \\
\text { ayudado a mejorar mi rendimiento }\end{array}$ & 2,7 \\
\hline & & & $\begin{array}{l}\text { Este proyecto de implementación de un aula } \\
\text { interactiva digital ha sido útil en la mejora de mi } \\
\text { rendimiento }\end{array}$ & 2,6 \\
\hline
\end{tabular}

En la tabla 4 se destacan los positivos comentarios de los estudiantes y la buena disposición respecto de la utilización del equipamiento en clases. Esto se confirma puesto que son los aspectos de mayor valoración para ellos: "Opinión del estudiante en relación a la eficacia de la enseñanza del profesor cuando se utiliza 
un aula interactiva digital" y "Apreciación y relación de los estudiantes con las nuevas tecnologías y el rediseño del programa de la asignatura". Así también se puede apreciar el aspecto de menor valoración: "Conexión entre el aprendizaje y la participación del estudiante cuando utiliza la pizarra interactiva digital, comparándola con el método tradicional de enseñanza pizarra y plumón", donde los comentarios de los estudiantes se enfocan que la pizarra interactiva debe ser utilizada en forma de apoyo y no necesariamente representa por si sola una buena clase.

Finalmente, este estudio se transforma de un modelo tradicional a un modelo innovador de docencia en Ingeniería, enfocado en asignaturas de especialidad de últimos niveles de una carrera netamente técnica.

\section{CONCLUSIONES}

Se genera gran expectación y ansias entre los estudiantes involucrados directamente en el estudio, en los profesores y funcionarios de la Unidad académica, tanto en la capacitación como uso de esta tecnología.

En particular, en la asignatura de Sistemas Térmicos se aprecia que las expectativas que tenía el curso respecto a la pizarra se lograron de manera exitosa, dado que los comentarios de los estudiantes fueron muy positivos, y hubo muy buena disposición acerca del uso de la pizarra en clases. Sin embargo, los estudiantes consideraron que la pizarra solamente debía ser utilizada como herramienta complementaria a los métodos de enseñanza tradicionales, dado que, hasta el momento, les parece más rápido y cómodo el uso de la pizarra clásica y el plumón.

En el caso de la asignatura de Formulación de Proyectos, se aprecia que el curso intervenido con la nueva metodología y tecnología presenta una tasa de rendimiento muy superior (93,35\%) al grupo control, el cual utilizó la metodología tradicional y no se usó tecnología en el aula, por ende se puede concluir que el uso de la tecnología y el cambio metodológico favorece el rendimiento de los estudiantes. Al comparar la tasa de aprobación entre ambos grupos estudiados, sin la prueba optativa de reemplazo, muestra que el uso del ADI favoreció el proceso de enseñanza aprendizaje en el curso más numeroso.

En términos generales, el presente estudio muestra una mejoría en el proceso de enseñanza - aprendizaje, sin embargo si se comparan las notas medias entre el grupo de control y experimental, éstas no muestran una diferencia significativa, lo que implica que la metodología debería ser perfeccionada en futuros estudios.

Respecto de la relevancia de este proyecto en el marco del Modelo Educativo Institucional, cabe señalar que se produce un pequeño cambio en la relación profesor y estudiante, originando una mejora continua en el proceso enseñanza - aprendizaje, donde la docencia se centra en los resultados del aprendizaje; de este modo, el estudiante se siente parte relevante de la construcción del conocimiento, evidenciándose una mayor responsabilidad en su propio aprendizaje.

\section{AGRADECIMIENTOS}

Los autores reconocen al Departamento de Ingeniería Mecánica y a la Vicerrectoría Académica de la Universidad de Santiago de Chile el apoyo prestado para este proyecto de Innovación Docente.

\section{REFERENCIAS}

Balmaceda, M., Malmod, A. y Díaz, V., Uso Pedagógico de las Nuevas Tecnologías: Indagación en el Potencial de la Multimedia como Herramienta Formativa, Congreso Internacional de Nuevas Tecnologías de Información y Comunicación, 34-40, Temuco, Chile, 19 a 20 de octubre (2006).

Cabero, J., La Aplicación de las TIC: ¿Esnobismo o Necesidad Educativa?, Red Digital: Revista de Tecnologías de la Información y Comunicación Educativas, ISSN: 1696-0823 (en línea), 1, (2000).

http://reddigital.cnice.mecd.es/1/firmas/firmas_cabero_ind.html. Acceso 7 de septiembre 2012

Candanedo, D.B., Educador del Siglo XXI, Formación Docente y las Tecnologías de Información y Comunicación, UNESCO, 139-144, (2005).

Cataldi, Z., Lage, F. y Denazis, J., Las TICS en la Educación Superior: Su Implicancia en la Enseñanza de Ingeniería, Congreso Internacional de Nuevas Tecnologías de Información y Comunicación, 261-269, Temuco, Chile, 19 a 20 de octubre (2006).

Gomes, P., El educador reflexivo: notas para la orientación de sus prácticas docentes, Educar, 30, 57-67 (2002). 
González, V., El Rediseño de Asignaturas Utilizando TICS, Congreso Internacional de Nuevas Tecnologías de Información y Comunicación, 239-245, Temuco, Chile, 19 a 20 de octubre (2006).

Guzmán, M., Hunter, R., Pérez, H. y Vizán, A., Adquisición Remota de Datos en un Proceso de Mecanizado, Congreso Internacional de Nuevas Tecnologías de Información y Comunicación, 255-260, Temuco, Chile, 19 a 20 de octubre (2006).

Martínez, P., Aprender y enseñar: Los estilos de aprendizaje y de enseñanza desde la práctica del aula, $1^{\mathrm{a}}$ edición, Mensajero, Bilbao, España (2007).

Prieto, L., La Autoeficacia del profesor universitario: Eficacia percibida y práctica docente, $1^{\mathrm{a}}$ edición, Narcea, Madrid, España (2007).

Rué, J., Enseñar en la Universidad: El EEES como reto para la Educación Superior, $1^{\mathrm{a}}$ edición, 163-185, Narcea, Madrid, España (2007).

Salinas, J.M., Cambios Metodológicos con las TIC: Estrategias Didáctica y Entornos Virtuales de Enseñanza-Aprendizajes, Revista de Orientación Pedagógica, 56(3-4), 469-481 (2004).

Villareal, G., La Pizarra Interactiva una Estrategia Metodológica de Uso para Apoyar la Enseñanza y Aprendizaje de la Matemática, Teoría de la Educación. Educación y Cultura en la Sociedad de la Información, ISSN: 1138-9737 (en línea), 1(7), (2006).

http://campus.usal.es/ teoriaeducacion/rev_numero_07/n7_art_villarreal.htm. Acceso: 10 de octubre 2009 
